\title{
EI REGISTRO PERIGLACIAR EN LA SIERRA DEL BARCO (SISTEMA CENTRAL) Y SU RELACIÓN CON EL SISTEMA GLACIAR PLEISTOCENO
}

\author{
RaQuel CRUz Ramos ${ }^{1}$ \\ Jose LuIs GoY GoY ${ }^{1}$ \\ CARIDAD ZaZO CARDEÑA ${ }^{2}$
}

\begin{abstract}
Resumen - El estudio geomorfológico llevado a cabo en la Sierra del Barco ha permitido reconstruir un número importante de formas y depósitos superficiales periglaciares, en relación con los procesos que las han originado y los dominios geomorfológicos en donde se han formado, obteniendo un inventario de formas específicas de este tipo de sistema. Se analiza su posible actividad actual y pasada y los ambientes de permafrost, nivación y ciclos de hielo/deshielo durante las etapas de formación. También se señala la secuencia cronológica relativa de estas formas en relación con el último ciclo glaciar, resaltando el efecto de los procesos periglaciares durante las fases glaciares de montera, máximo, valle, ladera y circo, así como la secuencia correspondiente al penúltimo ciclo glaciar y actualidad.
\end{abstract}

Palabras clave: Ambientes de permafrost, ciclos de hielo/deshielo, nivación, glaciares rocosos, suelos estructurados.

\begin{abstract}
The Periglacial ReCord in the Sierra del Barco (SISTEMA CenTRAL, SPAIN) AND ITS RELATIONSHIP WITH THE PLEISTOCENE GLACIAL SYSTEM. The geomorphological study carried out in the Sierra del Barco allow us to reconstruct a large number of periglacial features and deposits and relate them to the dominant causal geomorphic processes, thus obtaining an inventory of system specific features. Possible present-day and past permafrost, nivation and freeze-thaw processes during the stages of periglacial activity are analysed. The chronological sequence of these features relative to the Last Glacial stages is also presented. The effect of glacial processes during the "montera", maximum, valley, slope and cirque phases is pointed out, along with the sequence corresponding to the pre-Last Glacial and present.
\end{abstract}

Key words: Permafrost environments, freeze-thaw cycles, nivation, rock glaciers, patterned ground.

Recebido: 22/11/2007. Revisto: 03/04/2009. Aceite: 01/06/2009.

1 Dpto. Geología (Geodinámica). Facultad de Ciencias. Universidad de Salamanca. E-mail: rqcruz@usal.es, joselgoy@usal.es

2 Dpto. Geología, Museo Nacional de Ciencias Naturales. Universidad de Salamanca. E-mail: menze65@mnem.esic.es 
Resumo - Os vestígios PERIGlaciários na SERRA do BARCo (SISTEMA CENTRAL) E SUA RELAÇÃO COM O SISTEMA GLACIÁRIO PLISTOCÉNICO. O estudo geomorfológico realizado na Serra do Barco permitiu reconstituir muitas formas e depósitos superficiais periglaciários, bem como os processos que os originaram e os domínios geomorfológicos em que se desenvolveram. Depois de reconstituídas as formas específicas deste sistema, analisa-se a sua actividade actual e passada e os ambientes de permafrost, nivação e ciclos de gelo/degelo durante as etapas de evolução da paisagem. Assinala-se ainda a sequência cronológica destas formas, relacionando-as com a última glaciação, e evidencia-se o efeito dos processos periglaciários durante as fases glaciárias de montera, de máximo, de vale, de vertente e de circo, assim como a sequência das formas relativa à fase anterior ao último glaciário e à actualidade.

Palavras-chave: Ambientes com permafrost, ciclos de gelo/degelo, nivação, glaciares rochosos, solos estruturados.

\begin{abstract}
Résumé - Les marques périglaciaires dans la Sierra del Barco (CordiLHEIRA CENTRAL, ESPAGNE) ET LEURS RAPPORTS AVEC LE SYSTÈME GLACIAIRE PLÉISTOCĖNE. L'étude géomorphologique réalisée dans la Sierra del Barco a permis de mettre de nombreux dépôts et formes périglaciaires en rapport avec les processus qui les ont créés et avec les domaines géomorphologiques où ils se sont formés, et de faire l'inventaire des formes particulières à ce type de montagne. On a analysé leur possible activité actuelle et passée, ainsi que les milieux de permafrost, de nivation et de cycles gel/dégel, au cours de leurs étapes de formation. On situe aussi la succession chronologique de ces formes par rapport au dernier cycle glaciaire, en montrant l'impact des processus périglaciaires pendant les phases de montera, maximale, de vallée, de versant et de cirque, ainsi que la séquence correspondant à l'avant dernier cycle glaciaire et à l'actualité.
\end{abstract}

Mots-clés: Milieu de permafrost, cycles de gel/dégel, nivation, glaciers rocheux, sols structurés.

\title{
I. SITUACIÓN GEOGRÁFICA Y CONTEXTO GEOLÓGICO-GEOMORFOLÓGICO
}

La sierra del Barco con cota media de 2000 m y máxima de $2399 \mathrm{~m}$ en el Canchal de La Covacha, se sitúa en el sector centro-occidental de la Sierra de Gredos (Sistema Central), y queda limitada al Norte y Sur por las depresiones tectónicas de la Cuenca de Corneja, Cuenca del Tietar, y al Oeste por los Valles del Aravalle y Jerte (fig. 1). Muestra asimetría de vertientes que condiciona el clima, la dinámica de los procesos y distribución de la vegetación.

A nivel climático presenta, en la actualidad, un régimen de humedad elevado, en el que las vertientes orientadas al S y SW reciben mayores precipitaciones al verse favorecidas por su orientación respecto a los vientos dominantes durante las épocas de los frentes de lluvia (otoño-invierno). En cuanto al régimen térmico, estas vertientes meridionales son más cálidas por su mayor insolación (Santa 
Regina, 1987) y por la ausencia de los vientos del Norte, que sí ejercen su influencia en la vertiente septentrional.

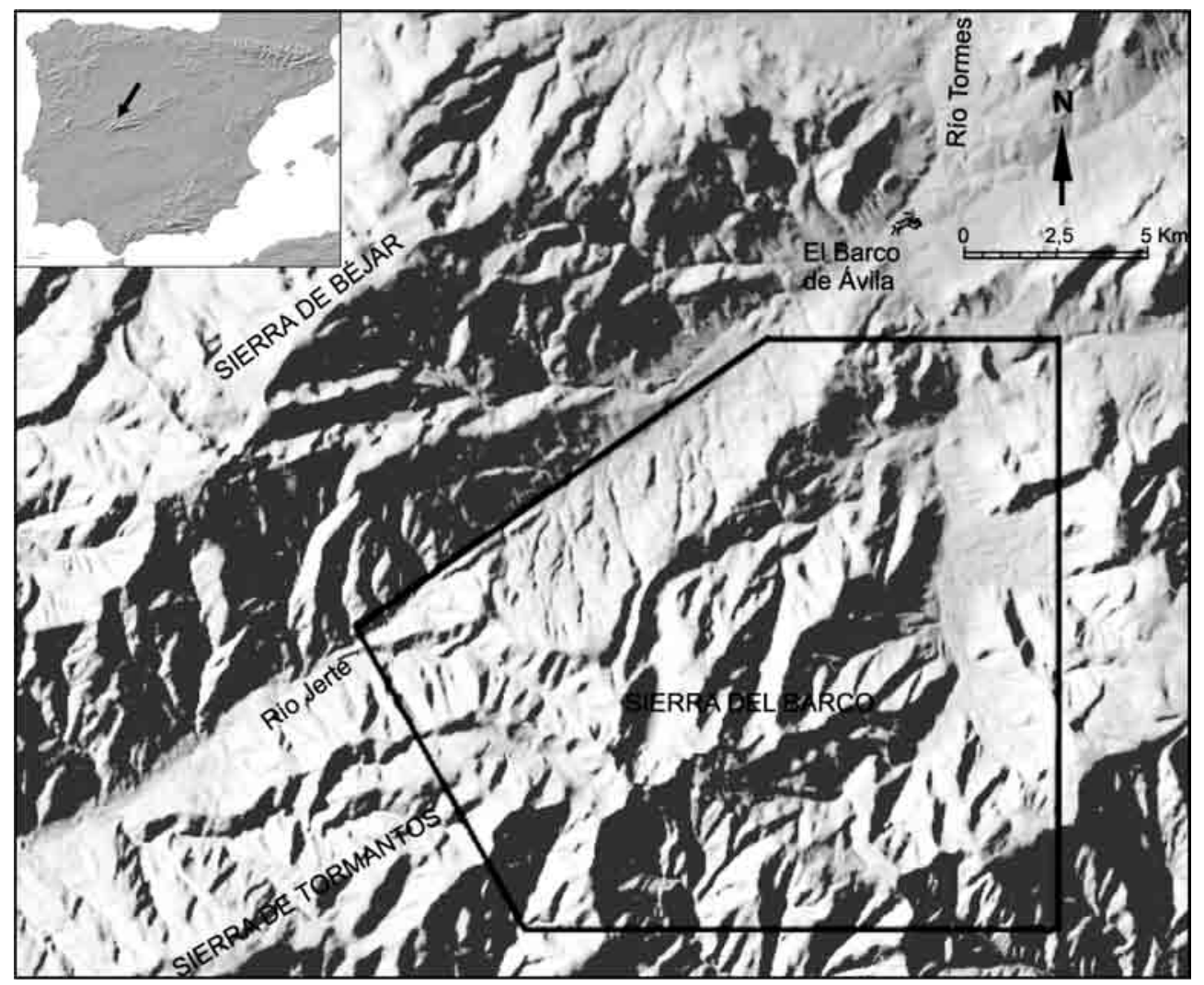

Fig. 1 - Localización del área de estudio.

Fig. 1 - Location of the study area.

A nivel geológico predominan las rocas ígneas con una importante alteración. Los granitos y migmatitas son las variedades predominantes, junto a los diques de cuarzo. Todo el sustrato se encuentra fracturado siguiendo sistemas NE-SW, NW-SE y N-S y E-W. Morfoestructuralmente es un macizo tectónico limitado por fosas, reactivado durante la orogenia alpina, consolidándose la superficie cimera en la posición actual durante el Plioceno superior/Pleistoceno (Pedraza, 1994); entendemos por ello que se vio afectado durante el Cuaternario por los cambios climáticos que se suceden (períodos fríos y cálidos), y dado que dentro de cada uno han existido oscilaciones de menor rango (subetapas y pulsaciones), consideramos que estos cambios pueden haberse registrado con mayor/ menor intensidad. 
La región se vio afectada por los hielos desde épocas anteriores al último ciclo glaciar, por lo que la morfogénesis glaciar ha sido muy activa, registrándose un número elevado de secuencias en circos y valles glaciares (Cruz et al., 2007). Este modelado ha sido objeto de numerosos estudios desde finales del siglo XIX (Casiano del Prado, 1864; Martín Donaire, 1879; Hernández Pacheco, 1902), y muy especialmente a partir de los años setenta (Martínez de Pisón y Muñoz Jiménez, 1973; Sanz Donaire 1976, 1977, 1982; Pedraza y López, 1980; Rubio, 1991; Rubio et al., 1992; Carrasco y Pedraza, 1995; Carrasco, 1999; Cruz et al., 2000; Cruz, 2006; Cruz et al., 2007). Por lo general, acompañando a este modelado, se hacen algunas referencias a la dinámica periglaciar, pero los primeros trabajos centrados en este sistema de una manera concreta no aparecen hasta los años noventa (Rubio, 1991; Carrasco, 1999; Cruz, 2006). Estos trabajos dejan un buen inventario, una cartografía precisa y la idea de un sistema muy activo en los momentos fríos del Cuaternario, que actuó como un importante agente modelador. El trabajo realizado desde entonces nos ha permitido ver la importancia que aún tienen esos procesos en el modelado, iniciándose estudios de formas y procesos activos, centrados en el análisis de la cobertura nival (extensión, distribución y duración), de los suelos helados estacionales (ciclos, duración, etc.) y en la posible evolución de las formas subactuales y actuales, que nos permitirán entender mejor los procesos y la dinámica periglaciar a nivel general.

\section{REGISTRO PERIGLACIAR. FORMAS Y FORMACIONES SUPERFICIALES}

En el estudio llevado a cabo se ha completado el inventario de formas y formaciones superficiales (F.S), que por su número indican la gran importancia que ha tenido la actividad periglaciar. En conjunto se distribuyen en un dominio que abarca desde las cimas hasta cotas de $1040 \mathrm{~m}$, aunque puede ser más amplio. La gran variedad, origen, estado y la colonización vegetal de muchas de ellas, nos hablan de ambientes periglaciares variados, relacionados con las diferentes etapas/subetapas/pulsaciones pleistocenas y oscilaciones del Holoceno. De las formas y F.S inventariadas, algunas son características de ambientes muy fríos (posiblemente permafrost), otras de ambientes de nivación y ciclos de hielo/deshielo intensos (Tabla I). 
Tabla I - Formas y formaciones superficiales del modelado periglaciar en la Sierra del Barco.

S - Superficies; L - Laderas; V - Valles; C - Circos.

Table I - Periglacial landforms and deposits of Sierra del Barco.

$S$-Summits, $L$-Slopes, $V$-Valleys, $C$ - Cirques.

\begin{tabular}{|c|c|c|c|c|c|c|}
\hline TIPO & AMBIENTE & PROCESOS & ESTADO & FORMAS & $\begin{array}{l}\text { EJEMPLOS } \\
\text { SIGNIFICATIV }\end{array}$ & \\
\hline \multirow{6}{*}{ 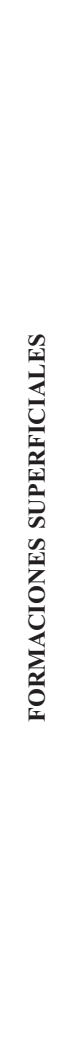 } & $\begin{array}{l}\text { Ciclos Hielo/ } \\
\text { Deshielo }\end{array}$ & \multirow[t]{2}{*}{$\begin{array}{l}\text { CRIOCLASTIA } \\
\text { Gelifluxión } \\
\text { Creep de helada } \\
\text { Solifluxión y lavado nival }\end{array}$} & $\begin{array}{l}\text { INACTIVAS/ } \\
\text { Semi- } \\
\text { funcionales }\end{array}$ & $\begin{array}{l}\text { Pedreras } \\
\text { Taludes y Conos } \\
\text { Campos, Laderas } \\
\text { y ríos de bloques }\end{array}$ & $\begin{array}{l}\text { Caballeror/San } \\
\text { Martín } \\
\text { Nava/Barco } \\
\text { Jaranda/Nava } \\
\text { Barco/serrada }\end{array}$ & $\begin{array}{l}\mathrm{V} \\
\mathrm{C} \\
\mathrm{L}, \mathrm{S}\end{array}$ \\
\hline & $\begin{array}{l}\text { De Nivación } \\
\text { Permafrost }\end{array}$ & & INACTIVOS & $\begin{array}{l}\text { Morrenas de névé } \\
\text { Glaciares Rocosos }\end{array}$ & $\begin{array}{l}\text { Hornillo/Serrada } \\
\text { San Martín } \\
\text { Barco/Covacha }\end{array}$ & $\begin{array}{l}\mathrm{L} \\
\mathrm{C}\end{array}$ \\
\hline & $\begin{array}{l}\text { Ciclos Hielo/ } \\
\text { Deshielo }\end{array}$ & $\begin{array}{l}\text { Segregación y } \\
\text { desplazamientos } \\
\text { en el suelo }\end{array}$ & $\begin{array}{l}\text { Activos } \\
\text { Estacionales }\end{array}$ & $\begin{array}{l}\text { Suelos } \\
\text { almohadillados } \\
\text { Hidrolacolitos }\end{array}$ & $\begin{array}{l}\text { Barco/Serra/ } \\
\text { Cuartos } \\
\text { Covacha/ } \\
\text { Infiernillos }\end{array}$ & $\begin{array}{l}\mathrm{V}, \mathrm{C} \\
\mathrm{V}\end{array}$ \\
\hline & $\begin{array}{l}\text { Permafrost } \\
\text { Ciclos Hielo/ } \\
\text { Deshielo }\end{array}$ & $\begin{array}{l}\text { LEVANTAMIENTO POR } \\
\text { LA HELADA } \\
\text { Agrietamiento por la } \\
\text { helada } \\
\text { Desplazamiento en suelo }\end{array}$ & INACTIVOS & $\begin{array}{l}\text { Suelos enlosados } \\
\text { Círculos de piedra } \\
\text { Bandeados/ } \\
\text { Estriados } \\
\text { Círculos de tierra }\end{array}$ & $\begin{array}{l}\text { Nava/Barco/Hoya } \\
\text { Mayor } \\
\text { Nava/Barco } \\
\text { Covacha/Cancho } \\
\text { Peña Cruz/Panera/ } \\
\text { Cardiel }\end{array}$ & $\begin{array}{c}\text { S } \\
\text { V, C } \\
\text { L, S }\end{array}$ \\
\hline & $\begin{array}{l}\text { Ciclos Hielo/ } \\
\text { Deshielo }\end{array}$ & \multirow{2}{*}{$\begin{array}{l}\text { MOVIMIENTOS } \\
\text { EN MASA }\end{array}$} & Activos & $\begin{array}{l}\text { Coladas solifluidales } \\
\text { y deslizamientos. } \\
\text { Bloques de arrastre }\end{array}$ & $\begin{array}{l}\text { Llano Redondo/ } \\
\text { Panera } \\
\text { Covacha/Cancho }\end{array}$ & $\begin{array}{c}\mathrm{L} \\
\mathrm{C}, \mathrm{S}\end{array}$ \\
\hline & Permafrost & & INACTIVOS & $\begin{array}{l}\text { Coladas gelifluidales } \\
\text { Mantos de } \\
\text { deslizamiento }\end{array}$ & $\begin{array}{l}\text { Caballeros } \\
\text { Lad. Espolón }\end{array}$ & V \\
\hline \multirow{2}{*}{ 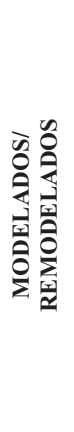 } & $\begin{array}{l}\text { Ciclos Hielo/ } \\
\text { Deshielo }\end{array}$ & $\begin{array}{l}\text { CRIOCLASTIA } \\
\text { NIVACIÓN (crioclastia, } \\
\text { gelifluxión, creep } \\
\text { de helada y aguas de } \\
\text { fusión) }\end{array}$ & $\begin{array}{l}\text { INACTIVOS/ } \\
\text { Semifuncionales }\end{array}$ & $\begin{array}{l}\text { Perfiles de } \\
\text { gelifracción } \\
\text { Nichos de nivación } \\
\text { Terrazas de } \\
\text { crioplanación }\end{array}$ & $\begin{array}{l}\text { Covacha/ } \\
\text { Infiernillos/ } \\
\text { Cuerda Mala } \\
\text { Hornillo/San } \\
\text { Martín } \\
\text { Cabeza Pelada }\end{array}$ & $\begin{array}{l}\mathrm{L} \\
\mathrm{S}\end{array}$ \\
\hline & $\begin{array}{l}\text { Ciclos Hielo/ } \\
\text { Deshielo } \\
\text { Ambiente de } \\
\text { Nivación }\end{array}$ & $\begin{array}{l}\text { CRIOCLASTIA Y } \\
\text { MOVIMIENTOS } \\
\text { EN MASA }\end{array}$ & INACTIVOS & $\begin{array}{l}\text { Valles disimétricos } \\
\text { Vertientes reguladas } \\
\text { Fondos de valle } \\
\text { plano }\end{array}$ & $\begin{array}{l}\text { Serrada/Caballeros } \\
\text { Los Piornales } \\
\text { Hornillo }\end{array}$ & $\begin{array}{l}\mathrm{V} \\
\mathrm{L} \\
\mathrm{V}\end{array}$ \\
\hline
\end{tabular}


La mayoría de las formas periglaciares se muestran inactivas pero algunas otras, a pesar de no estar conformes con las condiciones ambientales actuales, mantienen cierta funcionalidad (canchales, conos, suelos estructurados) y unas pocas son funcionales en las condiciones actuales (suelos almohadillados, hidrolacolitos, solifluxión, creep de helada, debris-flows). Posiblemente el sistema se haya mantenido activo durante gran parte del Cuaternario, si bien la mayoría de las formas que encontramos se desarrollaron en el último periodo glaciar (Cruz et al., 2006). La actuación durante todo este tiempo de diferentes procesos propios de los anteriores ambientes, definieron el dominio, caracterizado por una clara diferenciación por sectores, en relación con la influencia que ejercieron la orientación, la litología, la fracturación de los materiales y la topografía. La orientación cobró y aún cobra una gran importancia condicionando la sectorialización de ambientes y de procesos, en razón de su influencia sobre las características climáticas. La litología, la tectónica y las formas previas intervienen definiendo el tipo(s) de proceso(s) y su grado de desarrollo. También manifiesta superposición de formas en los sectores altos, en clara relación con la evolución climática a lo largo del Cuaternario.

En la tabla I quedan resumidos los tipos encontrados, correlacionándolos con ejemplos significativos, junto a los ambientes de formación y procesos que pudieron intervenir en su génesis y desarrollo, y el estado dinámico actual. Casi todos ellos se generaron por una combinación de procesos, en una misma época fría o en varias sucesivas, resultando por ello formas poligénicas. En la tabla II se expone la distribución encontrada de los diferentes tipos, en los distintos dominios geomorfológicos, relacionada con el ambiente/s que prevaleció en el momento de formación y con los factores anteriormente mencionados, que condicionaron el desarrollo de los procesos generadores.

\section{Formas y formaciones superficiales heredadas}

El registro muestra un amplio conjunto de formas generadas por la actuación continuada de procesos periglaciares y de procesos periglaciares y glaciares actuando conjuntamente. Encontramos buenos ejemplos de perfiles de gelifracción, con "agujas y torres" (relieves caracterizados por su morfología afilada), relacionadas con la acción diferencial a favor de líneas de fracturas, que indican la gran capacidad morfogenética que alcanzaron los ciclos de hielo/deshielo collados de transfluencia debidos a la fluencia de los hielos de una cuenca a otra durante la fase de máximo glaciar, terrazas de crioplanación relacionadas con procesos de nivación, recubiertas por till morrénicos, que habla de la importancia de la nivación en momentos previos a la glaciación (Tablas I y II, fig. 2), nichos de nivación en la vertiente de mayor insolación, con acumulaciones de clastos angulosos en su base, que junto a los valles asimétricos como los de Caballeros, Nava y Serrada hablan de la influencia de la orientación. El gran desarrollo que presentan estos valles, así como los de las formas y depósitos 

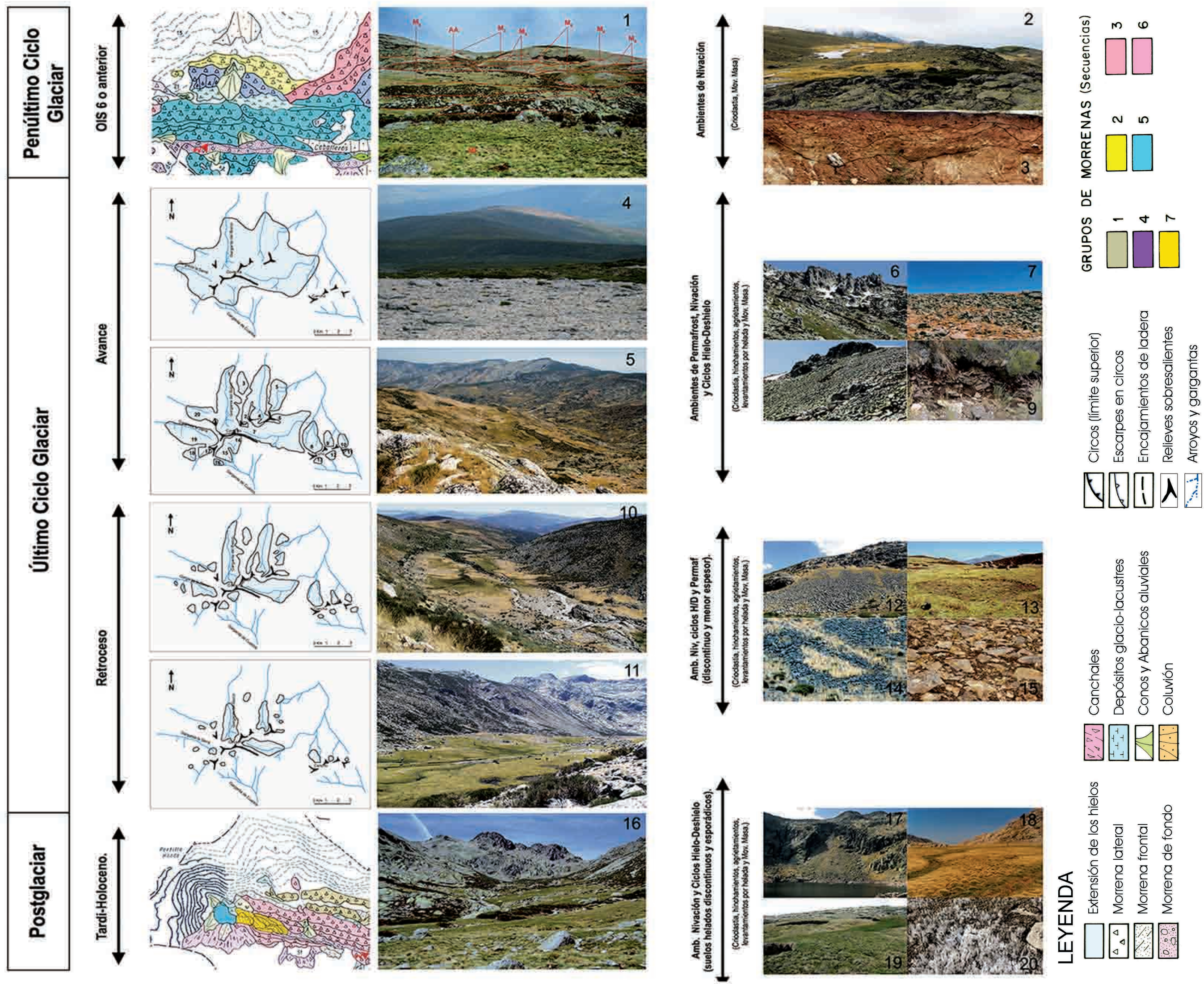
Tabla II - Localización, procesos, formas y formaciones superficiales periglaciares en la Sierra del Barco.

Table II - Location, processes, landforms and deposits of periglacial origin in Sierra del Barco.

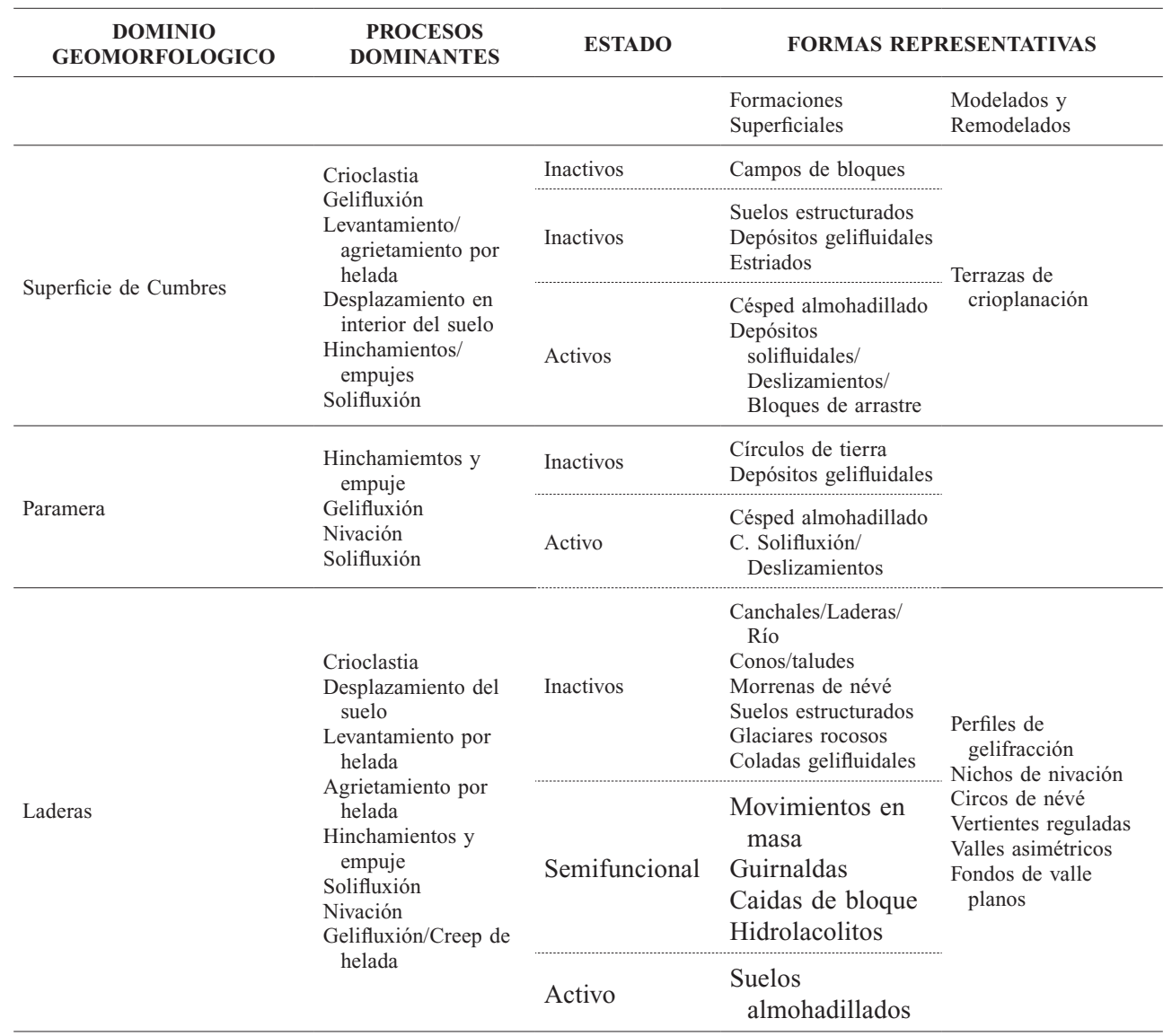

que albergan, hablan de un inicio temprano, que probablemente pueda centrarse en los primeros tiempos del empeoramiento climático.

También es rico el registro en Formaciones Superficiales heredadas, acumulaciones de materiales (en ocasiones bloques $>2 \mathrm{~m}$ en su eje mayor) resultantes de la removilización superficial, derivadas de procesos relacionados con la acción de ciclos de hielo-deshielo estacional, en ciertos casos asociados a la capa activa. Las más frecuentes son las extensas acumulaciones de gelifractos (pedreras, conos, taludes, laderas de bloques, glaciares rocosos, morrenas de néve,...) localizadas sobre superficies con distinta inclinación y que dan idea de la importancia de la crioclastia como proceso modelador. Igualmente son frecuentes los depósitos de gelifluxión (coladas y mantos de deslizamientos), que 
engloban bloques angulosos, en general caóticos, aunque en ocasiones muestran cierta estructuración, relacionados con movimientos en masa sobre las laderas, que han logrado regularizar algunos sectores, siendo indicativos de la importancia de estos procesos, probablemente en relación con el alto contenido en agua de la capa activa durante las épocas de deshielo. Además, cobran importancia, por ser indicadores de ambientes muy fríos, los abundantes suelos estructurados fósiles (polígonos, enlosados, estriados...) encontrados en zonas cimeras y partes altas de la sierra, (tablas I y II, fig. 2).

\section{Formas y formaciones superficiales actuales}

En la actualidad el sistema periglaciar se encuentra activo en sectores puntuales de ladera alta y superficies cimeras, entre noviembre y marzo, en cotas superiores a $1400 \mathrm{~m}$. Sus procesos, menos intensos que en épocas anteriores, están vinculados a la acción de la helada y de las aguas de fusión nival.

El análisis de campo permite confirmar la presencia de suelos helados estacionales durante buena parte de la estación invernal, que llegan a alcanzar relativa extensión en cotas superiores a los $1400 \mathrm{~m}$, localizándose en sectores cuya orientación y topografía no permite la acumulación de nieve, ni el desarrollo de vegetación. Estos suelos se establecen en periodos de altas presiones prolongados, tras épocas de bajas presiones y pueden superar la decena de centímetros de espesor, manteniéndose días, semanas y meses completos en relación con su exposición norte o sur y con las condiciones climáticas. La situación suele repetirse varias veces al año. También se configuran pavimentos nivales, en algunos sectores como en las superficies de Panera y Cancho, en relación con el levantamiento de cantos y bloques de pequeño/mediano tamaño. La presión que ejerce el horizonte superficial helado, al incrementar su volumen, sobre las aguas de sectores próximos, fuerza su movimiento hacia la superficie, generándose hinchamientos del suelo, que originan hidrolacolitos y céspedes almohadillados. Son muy frecuentes en las zonas turbosas del dominio glaciar/periglaciar, los hidrolacolitos siempre en cotas mayores de $1600 \mathrm{~m}$ y los suelos almohadillados desde cotas de $1400 \mathrm{~m}$. Buenos ejemplos son los que se desarrollan sobre los depósitos turbosos en los circos del Barco, Cuartos y Caballeros (foto 18, fig. 2). También se desarrollan, en otoño/invierno, a veces diariamente, "agujas de hielo" de 5-50 mm (las mayores en depósitos morrénicos de los circos de Nava y Barco), que van desagregando el suelo, con ayuda del lavado de materiales por las aguas de fusión (foto 20, fig. 2).

Los procesos de crioclastia se mantienen en las zonas altas de la sierra, en relación con la fusión nival primaveral, y son frecuentes en zonas de circo con acumulación de nieve (corredores y escalones en paredes) y en relieves superiores; los ciclos de hielo-deshielo diarios aportan gelifractos a los conos y taludes existentes en la base de los circos, que no muestran recubrimiento liquénico como se aprecia en los taludes y conos de los circos de la Nava y Serrada. 
Los movimientos en masa siguen siendo muy representativos, favorecidos por la existencia de parte de la cubierta alterítica y de otras formaciones superficiales con abundancia de finos (turberas, morrenas y coluviones), que facilitan su sobresaturación por aguas de fusión. Las morfologías que les identifican son las coladas solifluidales y los deslizamientos que se producen en ellas al superar la resistencia al cizallamiento, relativamente frecuentes en zonas altas de ladera, como en la superficie de Panera (foto 19, fig. 2), morrenas y canchales de valles glaciares con pendientes mayores de $30^{\circ}$, como ocurren en la Nava y Barco, y las coladas de bloques (debris-flow), procesos activos muy abundantes en el valle de la Nava relacionados con la fusión nival y/o intensas precipitaciones.

\section{EVOLUCIÓN DE LOS FENÓMENOS GLACIARES Y PERIGLACIARES}

Aunque los fenómenos glaciares y periglaciares se iniciaron en épocas tempranas del Cuaternario, los registros correspondientes son pocos y fragmentados y resulta difícil su interpretación (Cruz et al., 2007). En cambio son numerosos los del último periodo frío, por ello la evolución que sigue se centra en esta etapa.

El registro glaciar encontrado habla de una dinámica más compleja de la hasta ahora admitida, que sólo se centraba en morfologías de depósito de la última glaciación (Rubio, 1991; Carrasco, 1999); este registro nos habla de fases de avance y retroceso numerosas, que muestran independencia en los diferentes sectores en relación con la configuración y localización del circo glaciar. La buena correlación entre fenómenos glaciares y periglaciares y la secuenciación relativa de procesos y formas realizada en base al registro, nos permite establecer algunas características de las diferentes etapas y fases de desarrollo, que se sucedieron durante la última glaciación (fig. 2).

\section{Penúltimo ciclo glaciar (OIS 6 y/o anteriores)}

Los fenómenos glaciar y periglaciar quedaron establecidos con anterioridad al último ciclo glaciar, aunque no podemos precisar el momento exacto. Los registros glaciares encontrados, como morrenas fragmentadas, encajadas y con gran evolución morfológica (foto 1, fig. 2), y también algunas formas periglaciares de cimas y zonas bajas de laderas, relacionadas con ambientes de nivación, podrían corresponderse con este ciclo glaciar. En las superficies cimeras de Cabeza Pelada, Horco y Cancho, se desarrollaron las terrazas de crioplanación encontradas (foto 2, fig. 2) y en la zona baja de la Gta. de Los Caballeros, próxima a su desembocadura en el Tormes (Cuenca del Duero), un cono gelifluidal (de Navalonguilla) relacionado con la terraza 25-30 m, dispuesto sobre un sustrato gleizado, constituido por un aglomerado de bloques angulosos, rubefactado y meteorizado, con un suelo rojo bien desarrollado con cutanes de ilu- 
viación, que habla de una época más cálida y húmeda que la actual, con estacionalidad marcada, lo que nos lleva a suponer que es anterior al último interglaciar y que posiblemente sea del penúltimo ciclo glaciar (Oxigen Isotope Stage - OIS 6) (foto 3, fig. 2).

\section{2. Último ciclo glaciar (OIS 4, OIS 2)}

En términos generales, en este período puede hablarse de una fase de montera, citada ya por Carrasco y Pedraza (1995), Carrasco (1999), en la Sierra de Béjar y Barco, y por Acaso et al., (2007), en Gredos, a la que siguen tres fases importantes de estabilización, con otras oscilaciones menores cada una.

La asimetría pluviométrica y térmica determinó que las masas de hielo alcanzaran mayor extensión y desarrollo en las vertientes con mayor precipitación/menor termicidad que en las de mayor insolación/pendiente. A lo largo de este período los ambientes periglaciares fueron variando de acuerdo con las oscilaciones climáticas que se suceden (fig. 2).

\section{a) Etapa de avance glaciar. Fases de montera y máximo}

En los momentos de avance glaciar, tuvieron lugar intensos procesos erosivos y deposicionales, también periglaciares. En principio pudo configurarse una pequeña montera de hielo, recubriendo las cimas de la que sobresaldrían los relieves mayores a modo de nunataks; a ella corresponderían los restos de till glaciar encontrados en los retazos de la Superficie de Cumbres existentes. Su pequeña magnitud nos hace pensar en una fase de corta duración (foto 4, fig. 2). Esta se continuaría con un periodo frío y más húmedo en el que los hielos alcanzaron la máxima extensión, configurándose un paisaje alpino. Durante esta fase, dado el espesor y duración de los hielos, los fenómenos erosivos fueron muy intensos, lo que dio lugar a importantes deposiciones registradas en los grandes complejos morrénicos externos (foto 5, fig. 2).

En los relieves sobresalientes a los hielos, a su alrededor y en sectores con altitud insuficiente para el desarrollo glaciar, se soportaron ambientes periglaciares muy fríos, de nivación y ciclos hielos/deshielos, que desencadenaron una gran variedad de procesos periglaciares, dando lugar a numerosas formas y formaciones superficiales. Destacan, en relación con la importancia de los procesos de crioclastia, los campos de bloques, cresterias y pedreras bajas (fotos 6 , 7 y 8, fig. 2), los mantos de deslizamiento (foto 9, fig. 2), suelos estructurados e hidrolacolitos en relación con movimientos en masa, levantamientos y agrietamiento por helada.

\section{b) Etapa de retroceso glaciar. Fases de Glaciares de Valle y Ladera}

Durante la etapa de retroceso glaciar se sucedieron dos fases de estabilización importantes, con oscilaciones de mayor o menor magnitud que motivaron variaciones en la extensión y espesor de los hielos, sin que se alcancen las condiciones de máximo, y en la extensión e intensidad de los ambientes/procesos 
periglaciares. Destaca como característica esencial de esta etapa, la importancia que cobran la orientación y la topografía en el desarrollo de ambientes y procesos, hecho que contribuyó al mayor desarrollo de la asimetría de algunos valles y también en las vertientes del macizo.

Prosiguen los intensos procesos erosivos glaciares (la mayor fluencia de los hielos en estos momentos tuvo que ver en ello) desarrollándose muchas de las formas que encontramos en ese dominio; la estabilización de los hielos permitió el desarrollo de complejos morrénicos intermedios (fotos 10 y 11, fig. 2). En relación con el periglaciarismo, el suelo helado vio reducido su espesor/ extensión, y probablemente sería discontinuo, haciéndose predominantes los procesos de crioclastia sobre el sustrato y de crioturbación, hinchamientos, levantamiento, agrietamiento por helada en el interior del suelo. En relación con la crioclastia continuó el desarrollo de los campos de bloques y la exageración de las crestas en las partes altas, iniciándose el desarrollo de pedreras inscritas en los valles glaciares (foto 12, fig. 2). La acción del resto de procesos continuaría con el desarrollo de los suelos estructurados en los sectores anteriores (fotos 14, 15, fig. 2) y con el inició, en los sectores libres de hielo glaciar y ahora con posibles restos de suelo helado, del proceso de regularización de vertientes dada la acumulación de derrubios en las laderas y la abundancia de agua de fusión/ precipitación nival que pudo ponerlos en marcha. También la génesis de círculos de tierra, como los encontrados en El Cardiel y Panera, relacionados con hundimiento de algunas elevaciones dómicas tras la fusión del núcleo de hielo (foto 13, fig. 2).

\section{c) Postglaciar. Fase de Glaciares de Circo}

En los últimos momentos de la glaciación, con condiciones frías y secas (Jalut, 1974; Serrat, 1979), los hielos sólo pudieron existir en los circos con mayores cotas (Caballeros y Serrada). En el resto del territorio, la nieve caída no fue suficiente para el desarrollo de los hielos, incrementándose los procesos periglaciares.

En relación con la menor intensidad erosiva, se desarrollaron los pequeños complejos morrénicos encontrados en los circos de Caballeros y Serrada; en ellos se llegan a diferenciar bien hasta una decena de pequeños arcos indicativos de otras tantas oscilaciones climáticas durante ese periodo (foto 16, fig. 2).

Probablemente ya habría desaparecido casi todo el suelo helado, pudiendo quedar restos sólo en sectores idóneos. En general los ambientes derivados de ciclos de hielo-deshielo intensos serían los protagonistas con intensos y extensos procesos crioclásticos que aportaron cantidad de derrubios; en ocasiones éstos pudieron movilizarse por hielo intersticial dando lugar a glaciares rocosos embrionarios (derrubios movilizados por gelifluxión), citados en el glaciar del Barco (Rubio 1991) y en San Martín (Carrasco, 1999) y que nosotros encontramos en la vertiente de umbría de los glaciares de la Nava y de Cuartos. En los circos sin hielo glaciar, los derrubios quedaron estabilizados en las paredes formando los conos y taludes que pueden contemplarse en ellos (foto 17, fig. 2). También 
continuarían los movimientos en masa como resultado del flujo de los derrubios por el deshielo nival y el desarrollo de suelos estructurados en las cimas y partes altas de ladera.

En la actualidad el fenómeno está circunscrito a sectores puntuales en cotas superiores de $1400 \mathrm{~m}$, siendo activo durante los meses de noviembre a marzo, con procesos vinculados a la acción de la helada y de las aguas de fusión nival: la crioclastia está muy atenuada y restringida a cotas superiores a $1800 \mathrm{~m}$ y las segregaciones de hielo en el interior del suelo a cotas mayores de $1400 \mathrm{~m}$. Los movimientos en masa siguen así siendo muy representativos, favorecidos por la existencia de una cubierta alterítica o de otras formaciones superficiales con abundancia de finos (zonas turbosas), que favorecen la saturación por las aguas de fusión. Son reflejo de ellos los suelos almohadillados (foto 18, fig. 2), terracillas, coladas solifluidales con deslizamientos (foto 19, fig. 2), debris flow y coladas de bloques que se desarrollan en los circos, morrenas y en la superficie cimera.

\section{CONCLUSIONES}

Del registro periglaciar se concluye un inventario de formas, relacionadas con los procesos que las originan (los procesos fundamentales han sido la gelifracción y los desplazamientos en masa), grado de actividad y su presencia en los ambientes glaciar/periglaciar y en el dominio geomorfológico en donde se originan, y una "secuencia cronológica relativa" de los procesos glaciares/periglaciares, precisando que, en relación con el proceso periglaciar, en el inicio de las fases frías cuaternarias los procesos de nivación cobraron gran importancia, generando terrazas de crioplanación y conos gelifluidales.

Durante el retroceso de los hielos se produjeron variaciones en la extensión del dominio, en la intensidad/tipo de procesos y en las formas, de modo que el desarrollo de los derrubios de ladera, suelos estructurados, hidrolacolitos y coladas gelifluidales fue ascendiendo de cota, desde las zonas bajas de laderas y valles torrenciales, a los fondos de valles glaciares y laderas altas.

En los últimos momentos, el sistema se convirtió en el agente morfogenético fundamental de las partes altas de ladera y circos, con la formación en estos sectores de glaciares rocosos, taludes/conos de derrubios y suelos estructurados. En la actualidad los procesos aunque atenuados y circunscritos a sectores puntuales de cimas, cabeceras torrenciales y laderas altas, mantienen cierta capacidad modeladora.

\section{AGRADECIMIENTOS}

Este trabajo ha sido subvencionado por los Proyectos SA041A08 (JCyL), CGL 200504655 y 01336/BTE y CGL 2008-03998 y 04000/BTE. 


\section{BIBLIOGRAFÍA}

Acaso E, Centeno J, Moya-Palomares M E (2007) Indicios de un glaciar de montera (Ice Field) en el Alto Gredos. In Lario J, Silva P G (Eds) Contribuciones al estudio del Periodo Cuaternario Aequa, Ávila: 39-40.

Carrasco R M (1999) Geomorfología del Valle del Jerte. Lineas maestras del paisaje. Universidad de Extremadura, Cáceres.

Carrasco R M, Pedraza J (1995) Morfología glaciar de las cimas de la Sierras de Gredos: Montera de Hielo. In Reconstrucción de paleoambientes y cambios climáticos durante el Cuaternario, Centro de Ciencias Medioambientales, C.S.I.C: 227-237.

Cruz R (2006) Análisis geológico-ambiental del Espacio Natural de Gredos. Cartografia del paisaje e itinerarios geoambientales. Tratamiento y representación mediante SIG. Tesis Doctoral, USAL, Universidad de Salamanca.

Cruz R, Goy J L, Zazo C (2000) Localización, procesos y formas periglaciares en la Sierra de BéjarCandelario (S.Central). In Peña J L, Sánchez-Fabre M, Lozano, M V (ed.) Procesos y formas periglaciares en la Montaña Mediterránea. Instituto de Estudios Turolenses: 191-211.

Cruz R, Goy J L, Zazo C (2006) Las Sierras de Béjar y del Barco durante el Cuaternario. Glaciarismo y periglaciarismo. In Goy J L, Cruz R, Zazo C, Cabero A (Eds.) Libro Guía de la XII Reunión Nacional de Cuaternario. USAL, Ávila: 28.

Del Prado C (1998) Descripción física y geológica de la Provincia de Madrid. Facsímil de la Editada en Madrid. Imp Nacional, 1864. I.T.G.E, Madrid.

Hernández Pacheco E (1902) Sobre la existencia de fenómenos glaciares en el Norte de Extremadura. Bol. R. Soc. Hist. Nat., 2: 127-131.

Jalut G (1974) Evolution de la végétation et variations climatiques durant les quinze derniers millénaires dans l'extrémité orientale des Pyrénées. Revue Géographique des Pyrénées et du SudOuest: 298-312.

Martín Donaire F (1879) Descripción física y geológica de la Provincia de Ávila. Mem. Com. del Mapa Geológico de España, Imprenta Manuel Tello, Madrid: 297.

Martínez de Pisón E, Muñoz Jiménez J (1973) Observaciones sobre la morfología del Alto Gredos. Est. Geogr. 129:3-103.

Pedraza J (1994) Sistema Central. In: Geomorfología de España. Ed. Rueda: 81-90.

Pedraza J, López J (1980) Gredos: Geología y Glaciarismo. Obra Social de la Caja de Ahorros de Ávila, Ávila.

Rubio Campos J C (1991) Geomorfología y Cuaternario de las Sierras de Béjar y de la Nava. Sistema Central. Tesis Doctoral, Ed. U.C.M.

Rubio Campos J C, Pedraza J, Carrasco R M (1992) Reconocimiento de tills primarios en el sector central y occidental de Sierra de Gredos (Sistema Central Español). In López Bermúdez et al. (eds.) Estudios de Geomorfología en España, Sociedad Española de Geomorfología. Murcia: 413-422.

Santa Regina I (1987) Estimaciones de la radiación solar según la topografía salmantina. Dip. Salamanca.

Sanz Donaire J J (1982) El macizo glaciarizado de El Barco de Ávila (Provincias de Ávila-Cáceres). Cuadernos de Geografia de la U. Complutense, 1: 183-206.

Sanz Donaire J J (1977) El glaciarismo de la cara sur del macizo del Barco de Ávila. V Coloquio de Geografia, Granada: 41-47. 
Sanz Donaire J J (1976) El corredor de Béjar. Tesis Doctoral, Fac. de Geografía e Historia, U.C.M.

Serrat D (1979) Rock glacier morainic deposits in the eastern Pyrenees. In Schluchter C (ed.). Moraines and Varves. A.A. Balkema, Rotterdam: 93-100. 